\title{
Improving Our Vision of Nanobiology
}

\author{
Deborah F. Kelly ${ }^{1}$ \\ ${ }^{1 .}$ Virginia Tech Carilion School of Medicine and Research Institute, Roanoke, VA 24016, USA.
}

Understanding the properties of molecular machines is a common goal of biologists and engineers. As such, transmission electron microscopes (TEMs) are often used to directly view the intricate details of molecular entities at the nanoscale. Biological assemblies that are preserved under frozen-hydrated conditions permit us to peer into the nanoworld in a near-native environment. This form of specimen preservation can be accomplished by plunge-freezing samples at high velocity into liquid ethane, thereby embedding the starting material in thin films of vitreous ice (Figure 1A). Conventionally, amorphous carbon is the most popular support material used to prepare biological specimens for TEM imaging. Micron-sized holes engineered into these carbon films provide a suitably transparent background to visualize biological complexes while embedded in vitreous ice (Figure 1B). However, there remain many limiting factors in high-resolution imaging, such as specimen charging, beaminduced movements, and other noise-producing artifacts.

The revolution in EM phase-plates, direct electron detectors, and in-column energy filters offers premiere technology to record pristine images of weak-phase objects. Correspondingly, the next generation of specimen support materials must also be developed to best utilize these new tools. New materials are being produced worldwide, which present a prime opportunity to test alternative substrates for EM support films. Such alternative substrates include, but are not limited to, conductive titaniumsilicon metal glass (Ti88Si12) [1], silicon carbide (cryomesh) [2], graphene [3], and silicon nitride (cryo-SiN) [4]. One recent example is the use of cryo-SiN to tether active viral assemblies while preserving them for cryo-EM imaging (Figure 1C) [5]. Another major benefit of using alternative substrates, such as silicon nitride, is their versatile surface properties. By decorating microchips or other substrates with specific adaptor molecules, we can create new tunable devices. Tunable microchips have been recently used to capture and visualize native protein assemblies from the nuclear material of patient-derived cancer cells (Figure 2). Moreover, with these new tools in hand, the field is uniquely poised to elucidate molecular processes in the context of human disease. In this tutorial, I will discuss a variety of considerations toward optimizing the preparation of biological macromolecules, ranging from the biochemical to the specimen preservation level.

\section{References:}

[1] Rhinow, D. and W. Kuhlbrandt. Ultramicroscopy. 108 (2008), pp. 698-705.

[2] Yoshioka, C., Carragher, B., and C. S. Potter. Microsc. Microanal. 16 (2010), pp. 43-53.

[3] Russo, C. J., and L. A. Passmore. Nat. Meth. 11 (2014), pp. 649-652.

[4] Tanner, J. R., Demmert, A. C., Dukes, M. J., Melanson, L. A., McDonald, S. M., and D. F. Kelly. J. Anal. Mol. Tech. 1 (2014), pp. 1-6.

[5] Kam J. A., Demmert, A. C., Tanner, J. R., McDonald, S. M., and D. F. Kelly. Technology. 2 (2014), pp. $1-5$.

[6] Scheres, S. H. J. Mol. Biol. 415 (2012), pp. 406-418. 

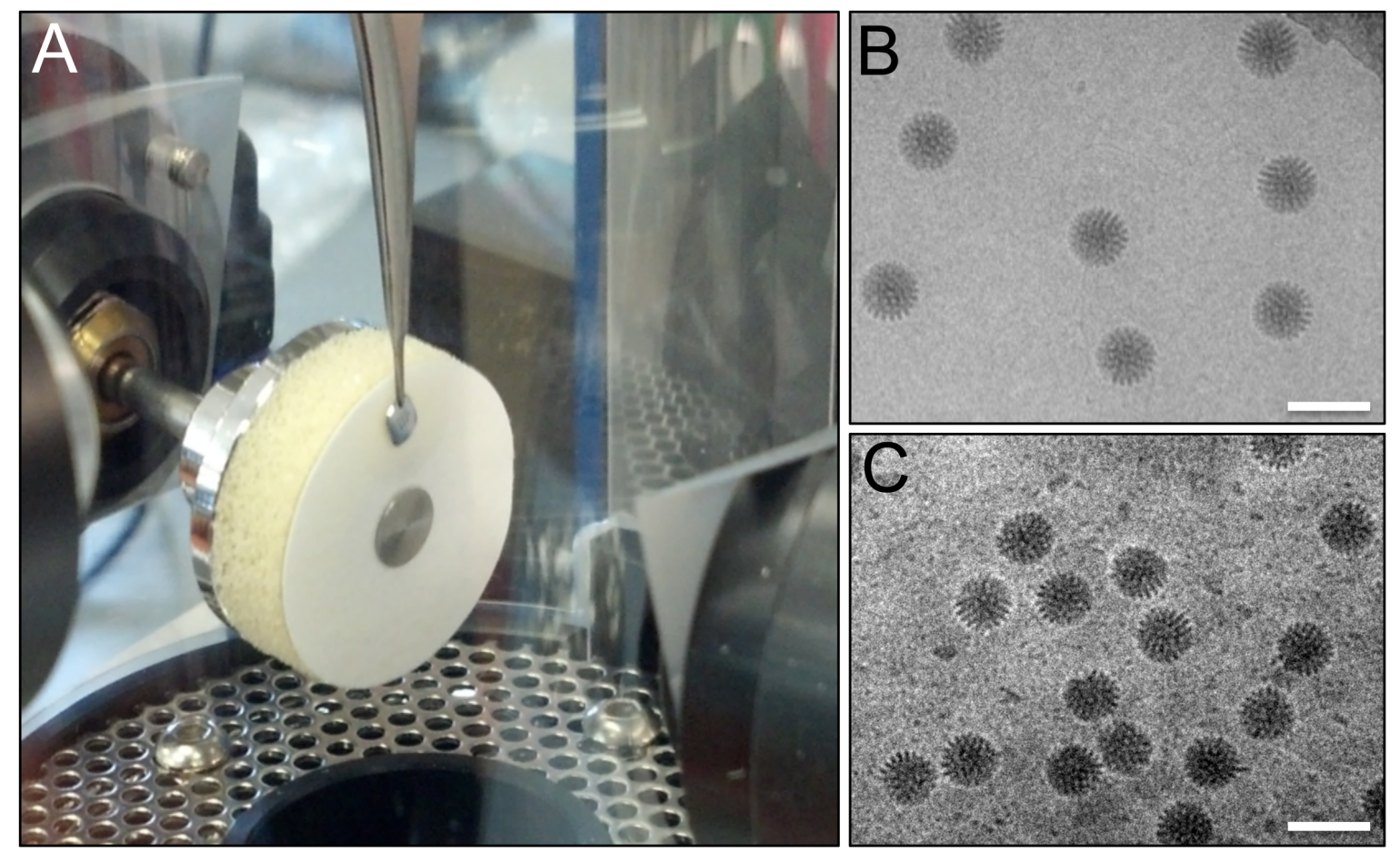

Figure 1. (A) Biological specimens are blotted and plunge-frozen into liquid ethane for cryopreservation and TEM imaging. A comparison of active rotavirus assemblies imaged over holey carbon support film (B) and optimized on cryo-SiN (C). Scale bar is $100 \mathrm{~nm}$.

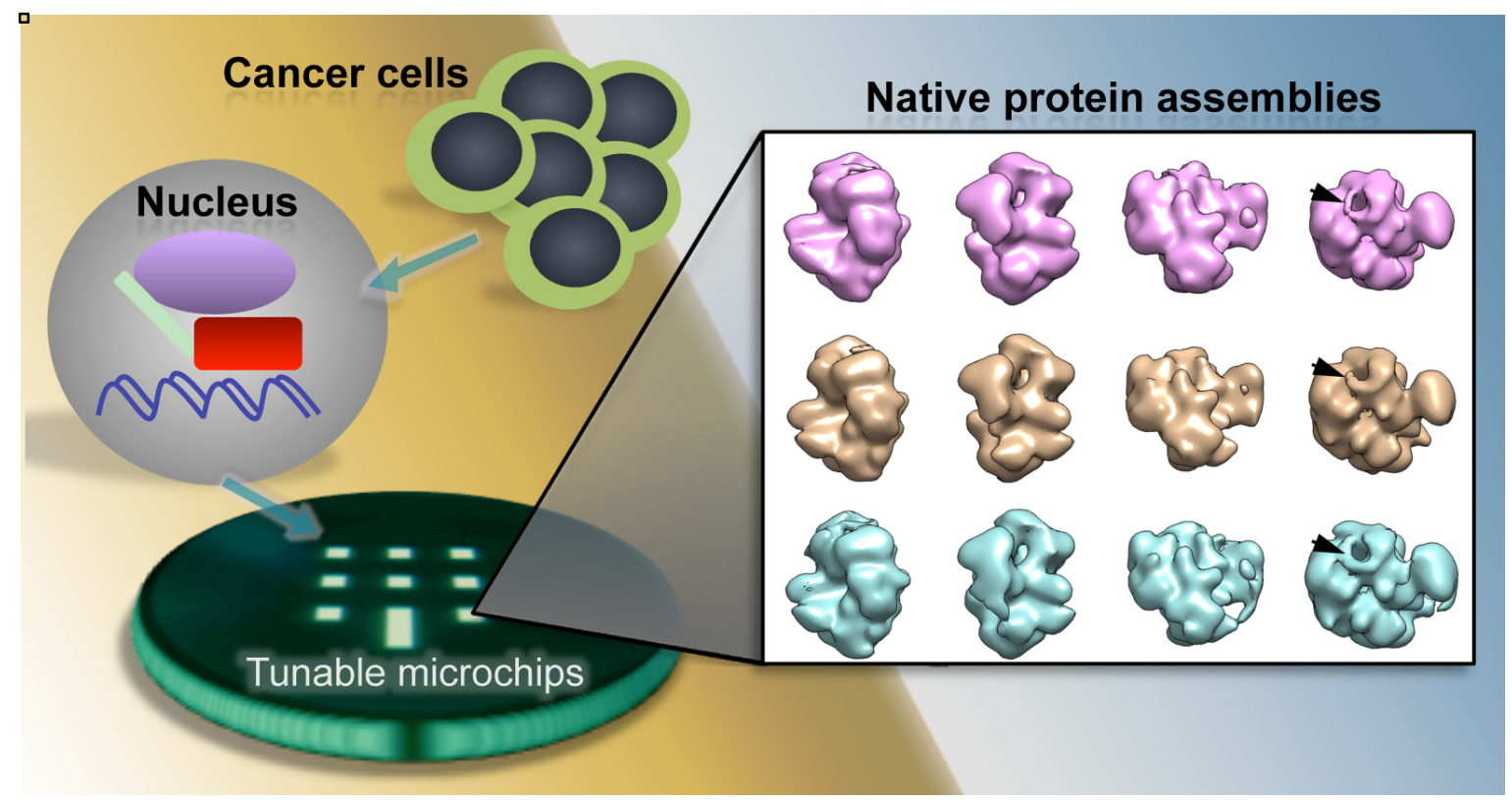

Figure 2. Native protein assemblies can be directly isolated upon tunable microchips from the nuclear material of patient-derived cancer cells. Captured assemblies were imaged using cryo-EM and reconstructed using 3D computing routines in the RELION software package [6]. 